4

Submitted to: Nuclear Instruments and Methods in Physics Research Section A: Accelerators, Spectrometers, Detectors and Associated Equipment

(1)

\title{
Neutron Angular Distribution in Plutonium-240 Spontaneous Fission
}

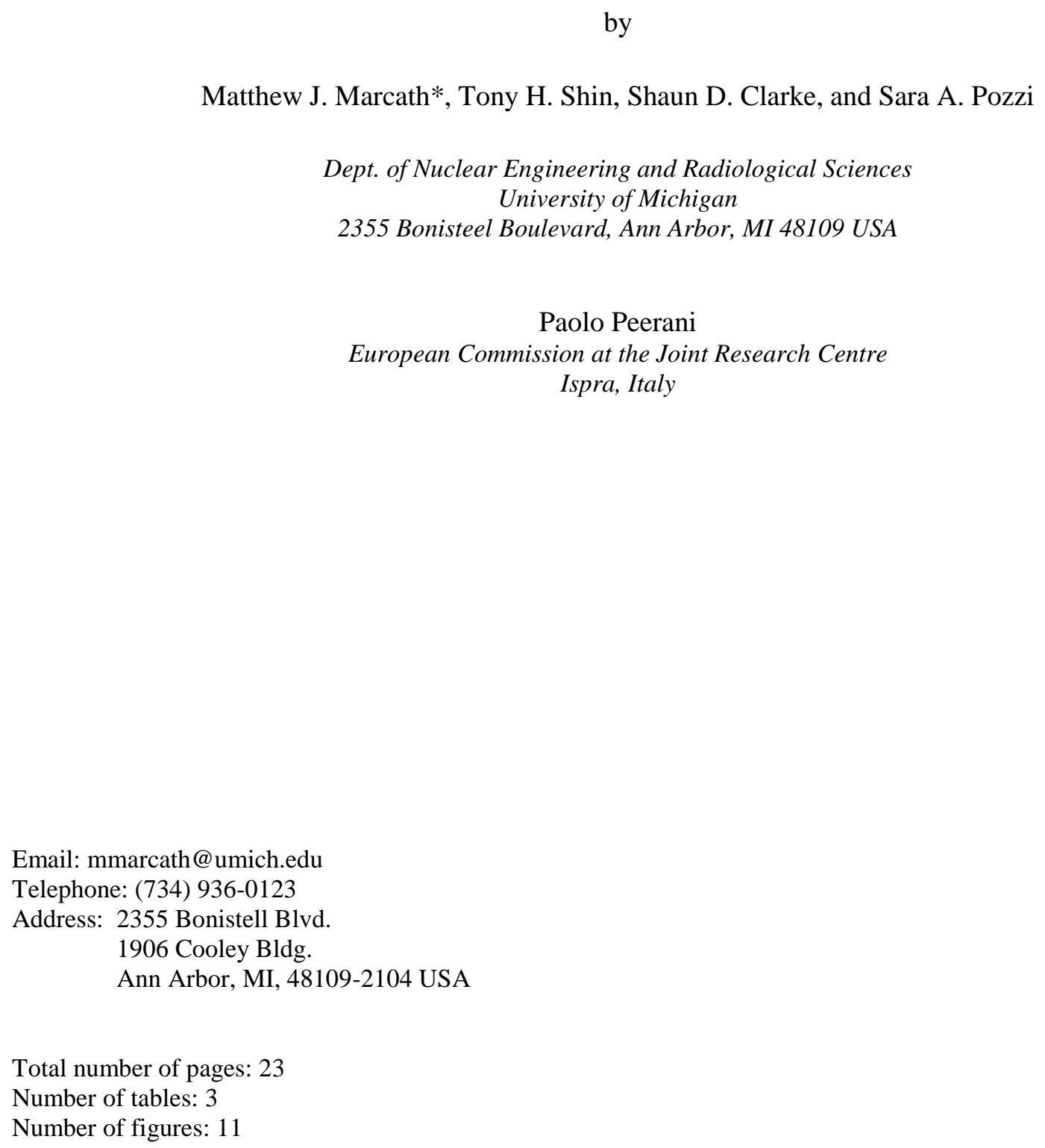

1

(C) 2016. This manuscript version is made available under the Elsevier user license http://www.elsevier.com/open-access/userlicense/1.0/ 


\title{
Neutron Angular Distribution in Plutonium-240 Spontaneous Fission
}

\author{
Matthew J. Marcath*, Tony H. Shin, Shaun D. Clarke, Paolo Peerani, and Sara A. Pozzi
}

\begin{abstract}
Nuclear safeguards applications require accurate fission models that exhibit prompt neutron anisotropy. In the laboratory reference frame, an anisotropic neutron angular distribution is observed because prompt fission neutrons carry momentum from fully accelerated fission fragments. A liquid organic scintillation detector array was used with pulse shape discrimination techniques to produce neutron-neutron cross-correlation time distributions and angular distributions from spontaneous fission in a ${ }^{252} \mathrm{Cf}$, a $0.84 \mathrm{~g}{ }^{240} \mathrm{Pu}_{\text {eff }}$ metal, and a $1.63 \mathrm{~g}{ }^{240} \mathrm{Pu}_{\text {eff }}$ metal sample. The effect of cross-talk, estimated with MCNPX-PoliMi simulations, is removed from neutron-neutron coincidences as a function of the angle between detector pairs. Fewer coincidences were observed at detector angles near 90 degrees, relative to higher and lower detector angles. As light output threshold increases, the observed anisotropy increases due to spectral effects arising from fission fragment momentum transfer to emitted neutrons. Stronger anisotropy was observed in Cf-252 spontaneous fission prompt neutrons than in $\mathrm{Pu}-240$ neutrons.
\end{abstract}

KEYWORDS: fission neutron anisotropy, plutonium, nuclear safeguards, MCNPX-PoliMi 
Plutonium is a special nuclear material that can be used in the core of a nuclear weapon. The odd-numbered isotopes of plutonium, in particular $\mathrm{Pu}-239$, are used to sustain a fission-chain reaction. However, bulk plutonium always contains some percentage of $\mathrm{Pu}-240$, which has a relatively high spontaneous fission rate. This feature makes plutonium detectable and quantifiable by passive means, and more specifically by counting neutron doubles [1]. In fact, fission generates multiple prompt neutrons emitted in coincidence. Over $70 \%$ of $\mathrm{Pu}-240$ spontaneous fissions emit more than one neutron [2].

Detection and characterization of special nuclear material often relies on knowledge of fission neutron anisotropy $[3,4,5,6]$. In fission, an anisotropic neutron angular distribution is observed in the laboratory reference frame because neutrons emitted isotropically in the fission fragment frame of reference carry momentum from the fully accelerated fission fragment $[6,7]$. Current nonproliferation and verification neutron measurement systems rely on detecting thermalized neutrons; the neutron properties at the time of emission are obscured by down-scattering. When fast neutron detectors are used, the neutron-neutron correlation from fission can be used to characterize fissile samples. Neutron-neutron correlation can distinguish metal from oxide and can estimate the fission to $(\alpha, \mathrm{n})$ rate [8]. In metals, fission neutrons are dominant, while in oxides, fission and $(\alpha, n)$ neutrons are both present. Fission neutrons are emitted anisotropically; $(\alpha, n)$ reactions, present in oxides, emit single neutrons, therefore neutron-neutron coincidences from $(\alpha, n)$ reactions are observed isotropically.

Many fission neutron angular correlation experiments have quantified anisotropy in fission neutron emission $[4,9,10]$; no published work exists, however, that investigates $\mathrm{Pu}-240$ spontaneous fission neutrons. Previous experiments characterize neutron correlations in $\mathrm{Pu}-239$ 
thermal neutron induced fission, but conclusions from this data are not directly applicable to $\mathrm{Pu}-$ 240 spontaneous fission [11].

Experimental results are presented here on correlated $\mathrm{Pu}-240$ spontaneous fission prompt neutrons, expanding on work performed by Dolan and colleagues [12]. Specifically, we present new results on the angular correlation of prompt neutrons from $\mathrm{Pu}-240$ fission. These experimental results are compared to simulations performed with the MCNPX-PoliMi v2.0.0 Monte Carlo code. Cross-talk coincidence effects are estimated with MCNPX-PoliMi simulations and are removed from the experimental neutron-neutron angular distributions.

\section{EXPERIMENT}

The experiment consisted of two plutonium experiments, using a $0.84 \mathrm{~g}$ and $1.63 \mathrm{~g}{ }^{240} \mathrm{Pu}_{\text {eff }}$ sample, hereby designated PM2 and PM3. A Cf-252 sample was also used. At the time of the experiment, the plutonium metal samples had fission rates of 400 and 760 fissions/s for PM2 and PM3, respectively; the Cf-252 sample had a fission rate of 26,000 fissions/s. The experiment was performed at the PERLA laboratory at the Joint Research Centre at Ispra, Italy [12].

Shown in Figure 2, 16-7.62 $\varnothing \times 7.62 \mathrm{~cm} \mathrm{EJ-309} \mathrm{organic} \mathrm{liquid} \mathrm{scintillation} \mathrm{detectors} \mathrm{were} \mathrm{used}$ to measure neutron-neutron coincidences from the plutonium samples. Two concentric 8detector rings were stacked; samples were placed along the central detector ring axis. The sample was shielded with a 1-cm thick lead cylinder to reduce the gamma-ray count rate and acquisition dead time. Each detector is approximately $18 \mathrm{~cm}$ from the central axis; the top and bottom detector rings are separated by $10.6 \mathrm{~cm}$ from detector centers. Two, time-synchronized CAEN V1720 digitizers were used to collect 120-sample pulses. Pulses above a 70-keVee threshold were recorded for offline processing. A 70-keVee acquisition threshold and a $2 \mathrm{~V}$ dynamic range used in this experiment correspond to a range of 0.65 to $6.7 \mathrm{MeV}$ proton recoils. 
Pulse shape discrimination by digital charge integration was used to discern neutron detections

110 from gamma-ray detections $[13,14]$. The pulse tail of a gamma-ray interaction decays faster than

111 a pulse of the same height from a neutron interaction. Waveforms that exhibit pile-up are

112 eliminated, and then a ratio of the pulse tail integral to the total pulse integral was used to

113 distinguish gamma-ray and neutron detections, shown in Fig. 1 for the PM2 sample. The PSD

114 line was assigned using an algorithm described by Polack and colleagues that minimizes particle

115 misclassification by analyzing segments of the tail-to-total integral points grouped by total

116 integral [15]. The photon to neutron ratio for this sample is approximately 100:1 making PSD

117 difficult at low pulse heights. The detection time differences of particles in a 60-ns coincidence

118 window were used to create cross-correlation distributions with 2-ns bins for each detector pair.

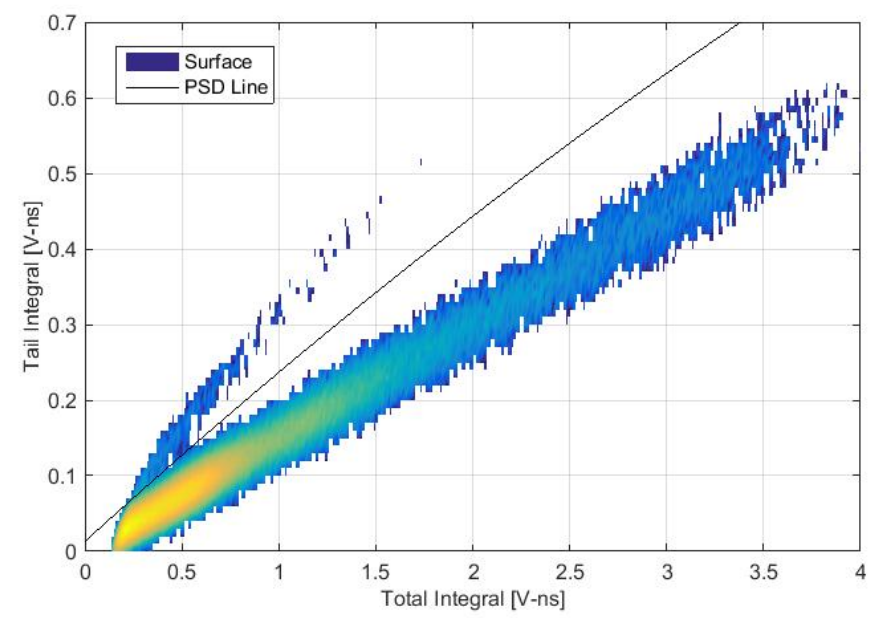

Figure 1: Experiment pulse shape discrimination plot of tail to total pulse integrals for 290,000

122 pulses from the PM2 sample. Color scaling is logarithmic ascending to yellow. Neutrons lie above the discrimination line. 
125 The MCNPX-PoliMi and MPPost codes were used to model the experiment geometry, the

126 plutonium metal sample, and the detector response [5]. The experiment assembly is shown in

127 Fig. 2a and the corresponding simulation model is shown in Fig. 2b. The detector

128 photomultiplier tubes, detector holder, aluminum table, and concrete floor were also modeled in

129 simulation, but were omitted from the figure for clarity.

130

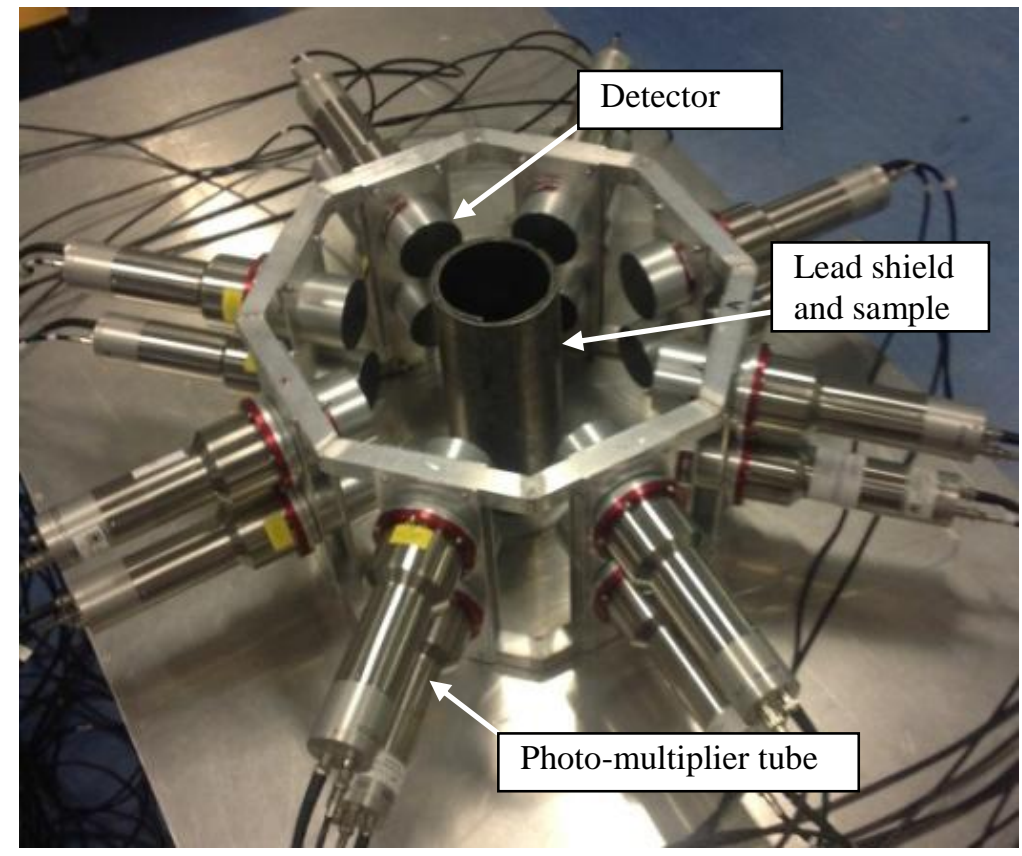

132 


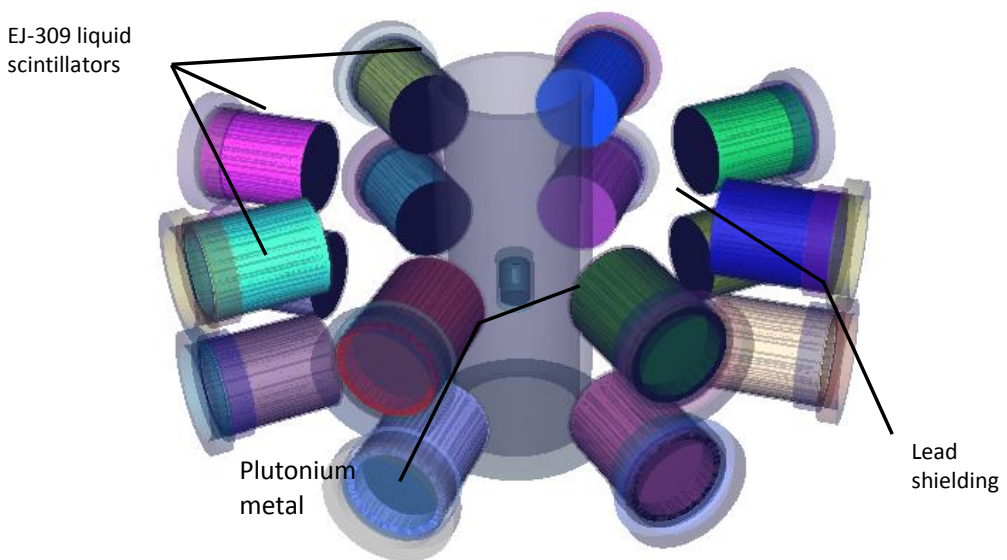

Figure 2: (a) A photograph of the experiment setup with a plutonium metal sample centered inside a lead shield and (b) the simulated plutonium metal experiment setup with EJ-309

$7.62 \varnothing \times 7.62 \mathrm{~cm}$ organic liquid scintillator detectors, plutonium metal, and lead shield are shown.

140 Each plutonium metal sample was modeled as a metal cylinder with composition described in 141 Tables I and II. The cylinder was topped by a void, and the void and cylinder were encased in 142 aluminum.

TABLE I. PLUTONIUM METAL SAMPLE ISOTOPIC COMPOSITION.

\begin{tabular}{|l|l|l|}
\hline Isotope & PM2 Weight Fraction & PM3 Weight Fraction \\
\hline Ni & 0.0504 & 0.0167 \\
\hline Cu & 0.0319 & 0.0427 \\
\hline Pu-238 & 0.00004 & 0.00023 \\
\hline Pu-239 & 0.87367 & 0.85141 \\
\hline Pu-240 & 0.04076 & 0.07891 \\
\hline Pu-241 & 0.00028 & 0.00089 \\
\hline
\end{tabular}




\begin{tabular}{|l|l|l|}
\hline Pu-242 & 0.00013 & 0.00044 \\
\hline
\end{tabular}

TABLE II. PLUTONIUM METAL SAMPLE PROPERTIES.

\begin{tabular}{|c|c|c|}
\hline Sample Property & PM2 & PM3 \\
\hline Total Mass [g] & 20.4 & 26.4 \\
\hline Density [g/cm $\left.{ }^{3}\right]$ & 19.7 & 22.4 \\
\hline${ }^{240}$ Pu Effective Mass [g] & $\mathbf{0 . 8 4}$ & 1.63 \\
\hline
\end{tabular}

The full isotopic composition of the samples was modeled in the material definition. Most 148 fissions are from $\mathrm{Pu}-240$ spontaneous fissions; only $0.5 \%$ and $0.9 \%$ of the spontaneous fissions are from $\mathrm{Pu}-242$ for the PM2 and PM3 samples respectively. Pu-238 spontaneous fission was

150 ignored. The PoliMi mixed-source option, $\operatorname{IPOL}(1)=99$, was specified to sample from $\mathrm{Pu}-240$ 151 and $\mathrm{Pu}-242$ built-in models. Using the plutonium metal sample MCNP model and the 152 spontaneous fission source term, it was estimated that approximately $8.4 \%$ and $10 \%$ of fissions are induced rather than spontaneous for PM2 and PM3. Also, approximately $88 \%$ and $79 \%$ of fission neutrons in the detectable range escaped the container and metal before interacting.

155 There are 120 possible detector pairings of 13 unique detector-sample-detector angles. Angles were calculated using the simulated mean neutron scatter position in the detectors for scatters 157 above the minimum detectable energy. Unique angles within 2 degrees of another were combined for 9 unique angle groups for PM2 and 10 groups for both PM3 and Cf-252, shown in

159 Table III. Variable sample position along the detector array central axis resulted in different 160 angle groups for each sample. Estimated angular uncertainty was determined by the neutron

161 scatter location variance, shown in Table III. The uncertainty varied for each angle grouping with 
162 the largest uncertainty being 13 degrees for the PM2 and PM3 cases and 18 degrees for the Cf-

163252 case. The average uncertainty in angle for each sample was 12 degrees, 11 degrees, and 13

164 degrees for PM2, PM3, and Cf-252 respectively.

TABLE III: DETECTOR-SAMPLE-DETECTOR ANGLES IN DEGREES FOR EACH SAMPLE POSITION.

\begin{tabular}{|c|c|c|c|c|c|}
\hline \multicolumn{2}{|r|}{ PM2 } & \multicolumn{2}{|r|}{ PM3 } & \multicolumn{2}{|c|}{ Cf-252 } \\
\hline Angle $\left[^{\circ}\right]$ & Uncertainty $\left[{ }^{\circ}\right]$ & Angle $\left[{ }^{\circ}\right]$ & Uncertainty $\left[^{\circ}\right]$ & Angle $\left[{ }^{\circ}\right]$ & Uncertainty $\left[{ }^{\circ}\right]$ \\
\hline 28 & 10 & 27 & 11 & 28 & 8 \\
\hline 42 & 12 & 41 & 11 & 44 & 15 \\
\hline 45 & 12 & 45 & 11 & 53 & 11 \\
\hline 52 & 12 & 51 & 12 & 87 & 14 \\
\hline 83 & 13 & 80 & 12 & 93 & 11 \\
\hline 91 & 13 & 91 & 13 & 127 & 14 \\
\hline 120 & 11 & 114 & 9 & 136 & 13 \\
\hline 135 & 11 & 132 & 10 & 149 & 12 \\
\hline 166 & 13 & 158 & 9 & 154 & 14 \\
\hline & & 175 & 11 & 177 & 18 \\
\hline
\end{tabular}

167 The MCNPX-PoliMi Pu-240 spontaneous fission neutron model includes a multiplicity-

168 dependent energy spectrum, multiplicity from zero to six, and anisotropy. Neutron angular

169 distributions relative to the light fission fragment direction for $\mathrm{Cf}-252$ and $\mathrm{Pu}-240$ are shown in

$170 \quad$ Fig 3. 


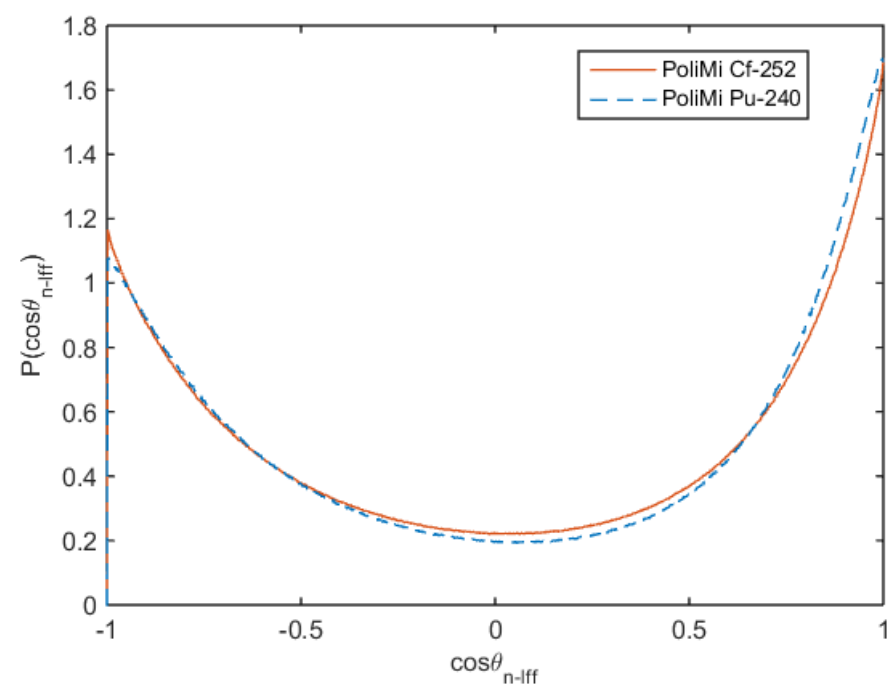

172

173

175

176

177 Experiment PM3 pulse height and time cross-correlation distributions are compared to

179 neutron coincidences versus detector pair angle for both experiment and simulation were

180 compared and are followed by subtraction of cross-talk coincidences from the experiment

181 results.

182

\subsection{Simulation Model Validation}

183 Simulated and experimental neutron pulse height distributions, shown in Fig. 4, for the top and 184 bottom rings of detectors were histogrammed. Two detectors in the array were removed from the

185 results due to inconsistent pulse height responses. Differences between the integral counts for the 186 top and bottom ring distributions are due to the samples placement slightly below the center 
187 plane of the detector array. The simulated pulse height distribution under-predicts the experiment

188 result by approximately $20 \%$ point-by-point over the dynamic-range, with exception at the

189 lowest pulse height bin. Gamma-ray misclassification increases toward lower pulse heights and

190 is likely the source of larger disagreement at low pulse heights. Consistent under-prediction

191 suggests that the aged fission rate used in the simulation is low. Improved agreement, with a

192 single detector and lower gamma-ray to neutron ratio, was demonstrated in work by Pozzi and

193 colleagues [16] when using a $3.3 \mathrm{~cm}$ lead shielded PM3 sample and a 7.62Ø×5.1 cm EJ-309

194 detector.

195

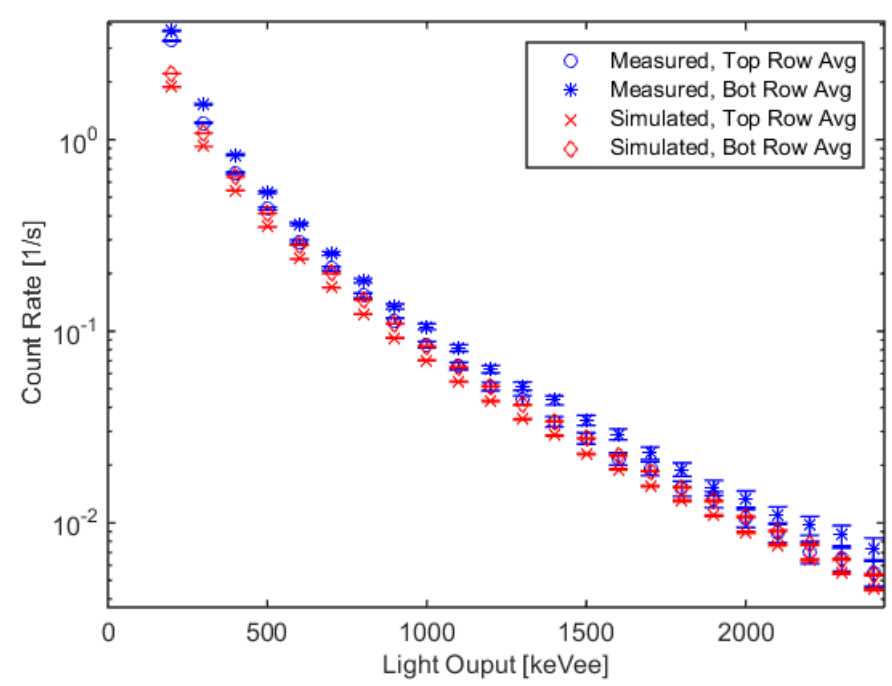

197 Figure 4: Experiment and simulated PM3 neutron pulse height distributions with a 100 keVee 198 threshold for the average of the top ring and the bottom ring. One standard deviation statistical error bars are shown. 
Overall, the MCNPX-PoliMi and MPPost models adequately reproduce experiment pulse

202 height distributions. Underestimation of the singles neutron rate does not impair the ability to

203 model double coincidences including trends in neutron-neutron time or angular correlations.

204 The PM3 time cross-correlation distributions from simulation agree well with the experiment 205 in shape for the 90-degree detector pair (Fig. 5a) and for the 175-degree detector pair (Fig. 5b).

206 Results show that there are fewer counts in the 90-degree detector pair distribution than the 175-

207 degree pair distribution because of the strong anisotropy in neutrons from spontaneous fission.

208 The simulation result slightly underestimates the experiment count rate for the 175-degree case.

209 Uncertainty in the plutonium sample position and incorrect neutron anisotropy models in 210 simulation contribute to disagreement in Fig. 5b. The distribution agreement in Fig. 5a and 211 attributed disagreement in Fig. 5b validates the MCNPX-PoliMi and MPPost models used to 212 represent the laboratory environment and detector response.
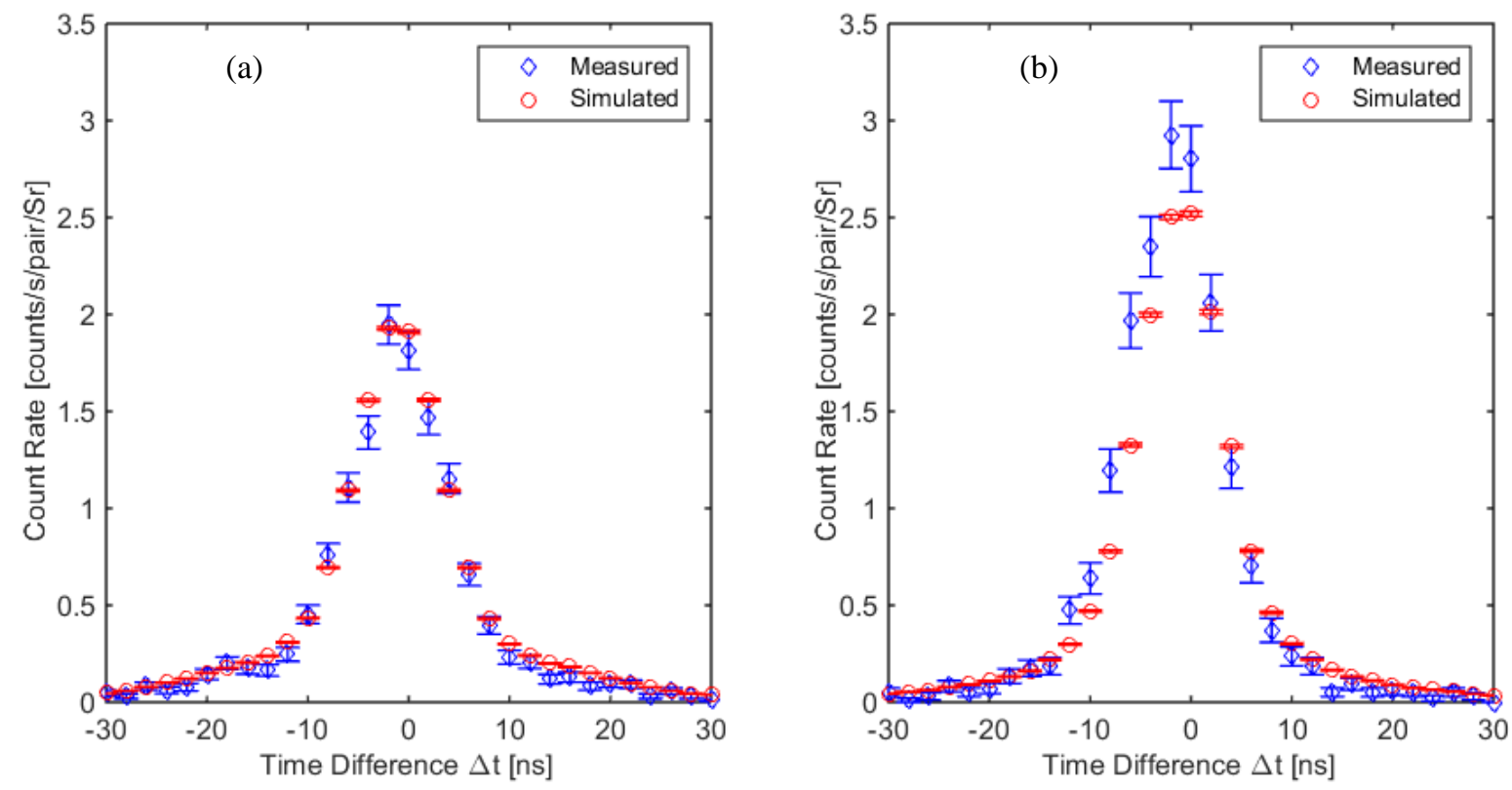
Figure 5: Experiment and simulated (MCNPX-PoliMi) PM3 neutron-neutron cross-correlation time distributions for 90-degree (a) and 175-degree (b) detector pairs with one standard deviation statistical uncertainty error bars.

For each unique angle, the neutron-neutron cross-correlation time distribution was integrated over a 60 ns window to give the total coincidence count rate, shown in Fig. 6. Each neutronneutron coincidence point is normalized by solid angle, detector pairs, and fission rate. Figure 6 highlights that a neutron is more likely to be emitted at small angles or very large angles relative to another neutron than to be emitted at angles around 90 degrees.

The MCNPX-PoliMi distributions in Fig. 6 match general features of the experiment distribution. The simulation underestimates the experiment count rate at angles larger than approximately 50 degrees, while the simulation overestimates at angles smaller than 50 degrees. The MCNPX-PoliMi fission model has too many low angle coincidences relative to the experiment result. The simulation result with cross-talk removed shows better agreement over a larger range of angles with the experiment result; cross-talk events are easily identified and removed in simulation space. The cross-talk removed results show that many low angle coincidences are from cross-talk and the cross-talk fraction diminishes toward large angles. 

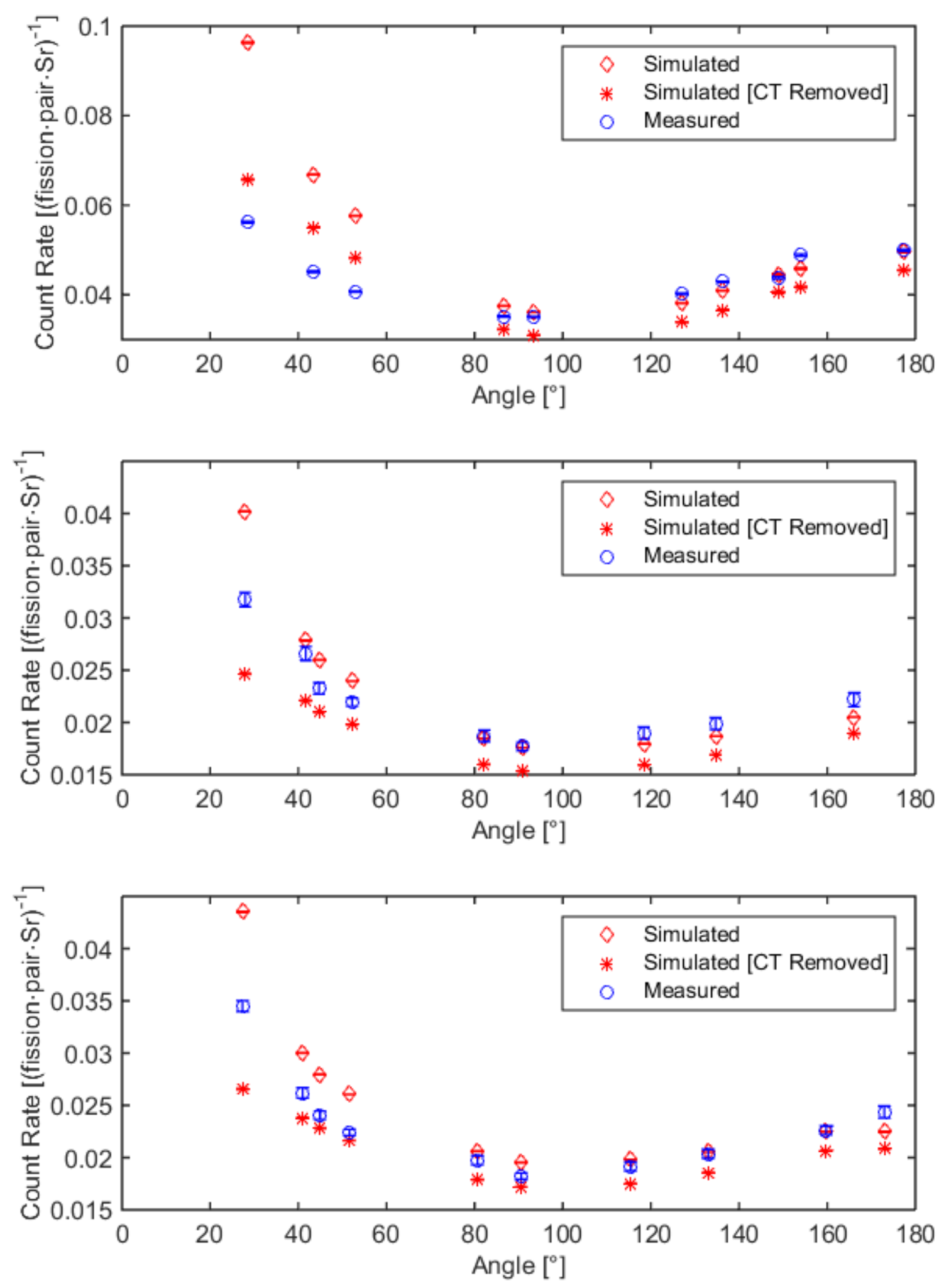
Figure 6: Simulated and experiment Cf-252 (top), PM2 (middle), and PM3 (bottom) neutronneutron coincidence angular distributions with a $70 \mathrm{keVee}$ threshold. Simulated results are shown with and without cross-talk. Vertical error bars represent one standard deviation statistical uncertainty; simulated vertical error bars are smaller than the symbols.

\subsection{Cross-talk Effect}

Cross-talk occurs when a single neutron is detected by more than one detector, mimicking a true coincidence. The probability of cross-talk for a given neutron spectrum and detector array can be reduced by inter-detector geometric attenuation, by shielding, or by an increased detection threshold. Inter-detector geometric attenuation or increased thresholds are not feasible, because the true coincidence count rate would also be reduced. The cross-talk fraction is reduced as the light output threshold is increased because the once-or-more scattered, lower-energy neutron falls below threshold more often than a direct, unscattered source neutron. Shielding attenuation was avoided in this work to minimize in-scattering of neutrons.

There is no reliable analysis for this experiment that could isolate cross-talk counts on an eventby-event basis, thus the simulated data were used to remove integral cross-talk counts from the experimental coincidences. The simulation collision history file allows the user to identify multiple detections that arise from a single neutron.

The simulation results in Fig. 7 show the number of cross-talk counts divided by the total number of coincidence counts. The 100, 150, and $200 \mathrm{keVee}$ thresholds correspond approximately to $0.8,1$, and $1.2 \mathrm{MeV}$ proton recoils, respectively. At the lowest angles for all thresholds, above $20 \%$ of coincidences are from cross-talk. Adjacent detectors share a large solid angle with each other and the cross-talk is correspondingly high, whereas opposing detector pairs near 180 degrees have cross-talk fractions under $10 \%$. 

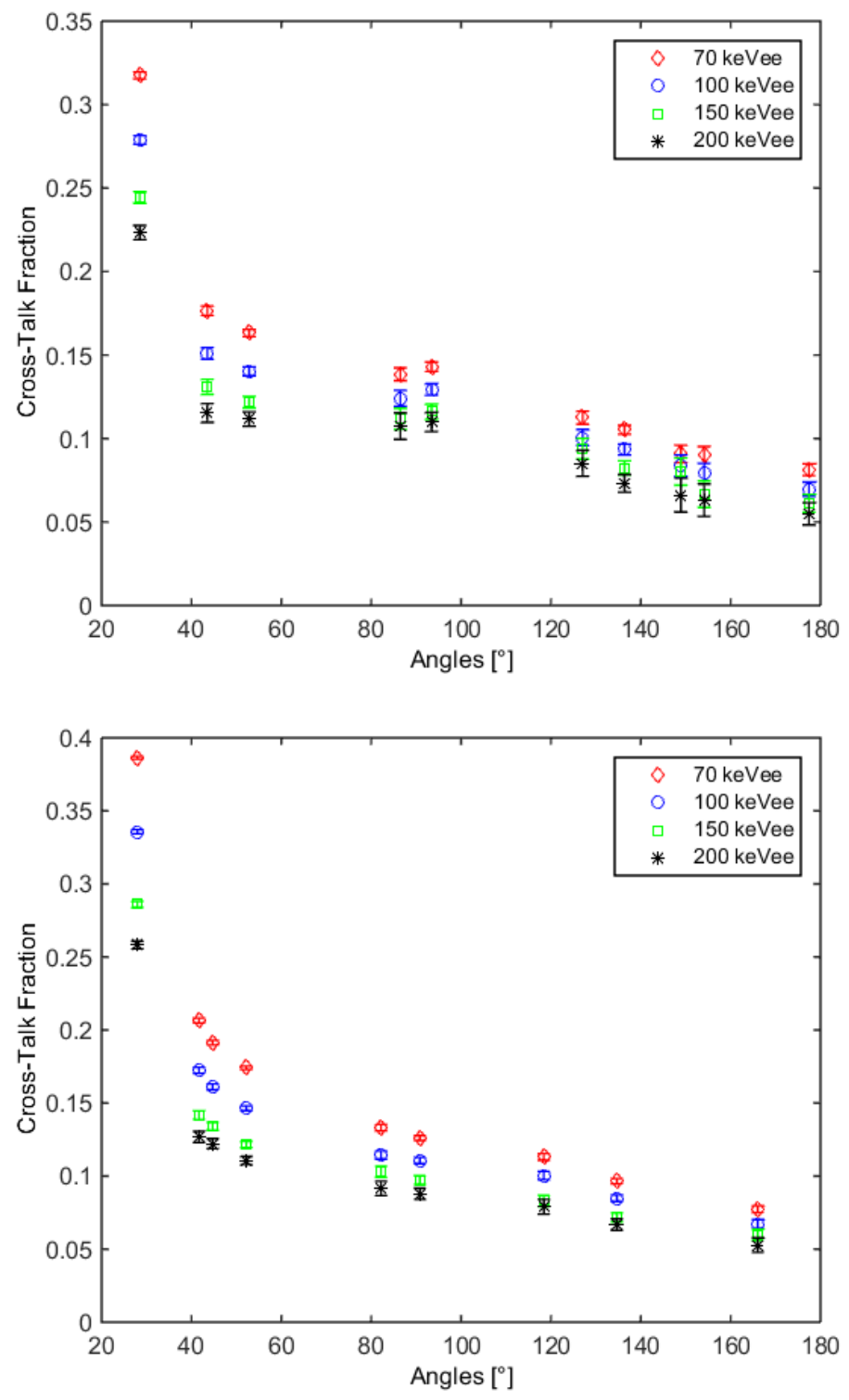


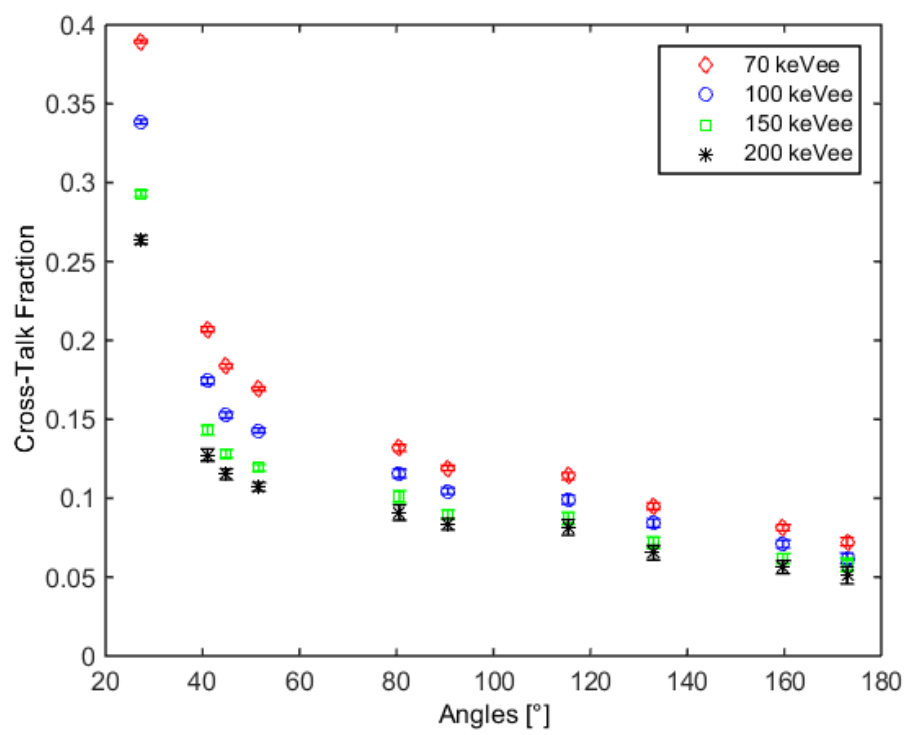

Figure 7: Simulated cross-talk fraction of total coincidences angular distributions for various

262 light output thresholds at each detector angle for Cf-252 (top), PM2 (middle), and PM3 (bottom).

\subsection{Cross-talk corrected angular distributions}

264 Figures 8-10 show the experiment neutron-neutron angular coincidence distribution after crosstalk removal. Experiment geometry specific effects in the angular distributions are minimized when coincidences from cross-talk are removed. The simulated cross-talk fraction is reliable

267 because it is primarily dependent on well-modeled factors, the detector array geometry and the 268 incident neutron energy spectrum. The MCNPX-PoliMi cross-talk removed result in Fig. 6 has a 269 higher count rate at low angles relative to those near 180 degrees, contrary to the trends observed 270 in Figs. 8-10. Therefore, the MCNPX-PoliMi model could be improved.

271 In Fig. 8, the Cf-252 coincidence distributions are compared to work by Petrov and colleagues 272 [17]; the Petrov experiment had a $50 \emptyset \times 50 \mathrm{~mm}$ and a $40 \emptyset \times 60 \mathrm{~mm}$ stilbene crystal $50 \mathrm{~cm}$ from a 273 Cf-252 source. This work compares well qualitatively with the Petrov data set. Differences 274 between the two distributions are attributed to better angular resolution in the Petrov experiment. 
275 For all samples, the neutron-neutron angular distributions with cross-talk coincidences removed 276 show that low angle coincidences are less likely than those at higher angles. Also, the neutron277 neutron angular distribution becomes more anisotropic as the pulse height threshold increases.

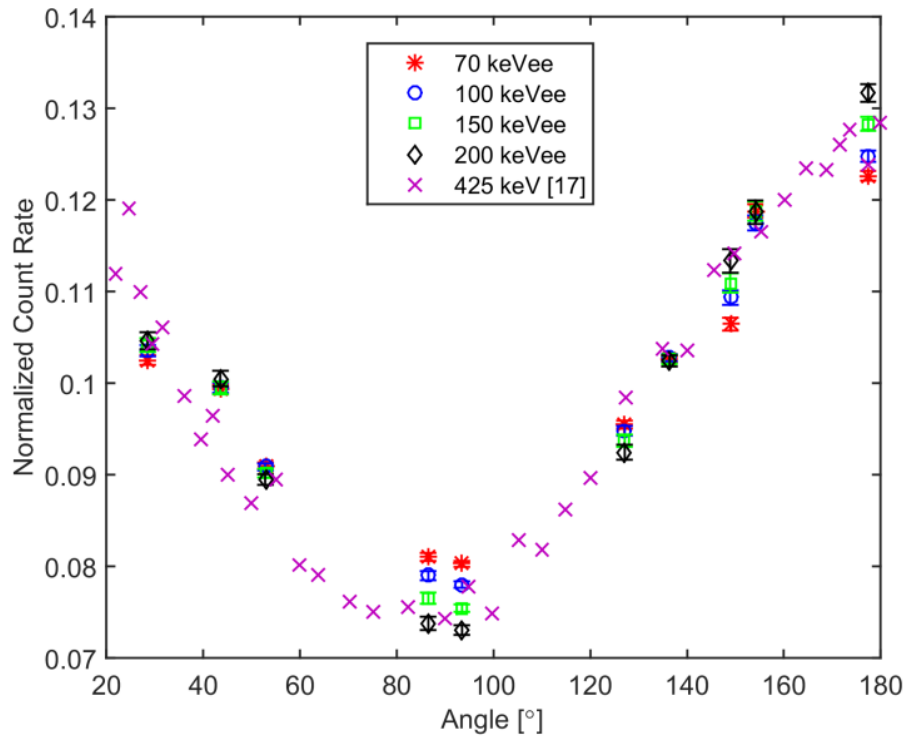

Figure 8: Experiment Cf-252 neutron-neutron cross-talk corrected coincidence angular distributions with a light output threshold varied over 70 to $200 \mathrm{keVee}$; experiment data are compared to data normalized by integral and number of points from Petrov et al. (X's) [17]. Vertical error bars represent one standard deviation statistical uncertainty.

284 The coincidence data point for PM2 in Fig. 9 at 42 degrees is higher than the point at 28 285 degrees likely because of sample position uncertainty, which affects the simulation estimated 286 cross-talk. At low detector angles, the contribution of cross-talk is very sensitive to small 287 changes in the sample position. 


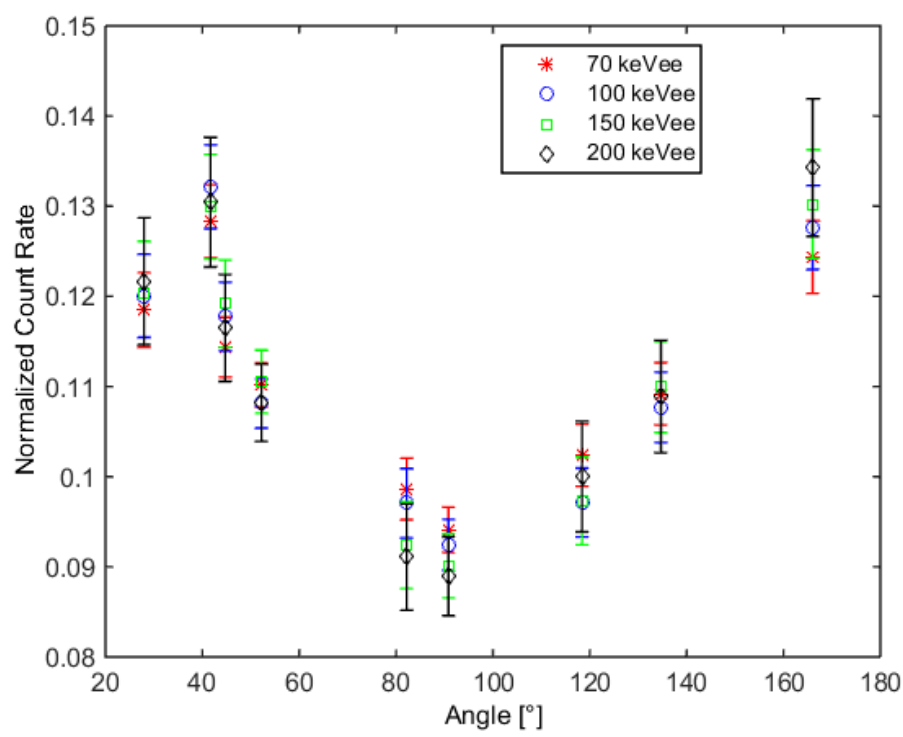

Figure 9: Experiment PM2 neutron-neutron cross-talk corrected coincidence angular

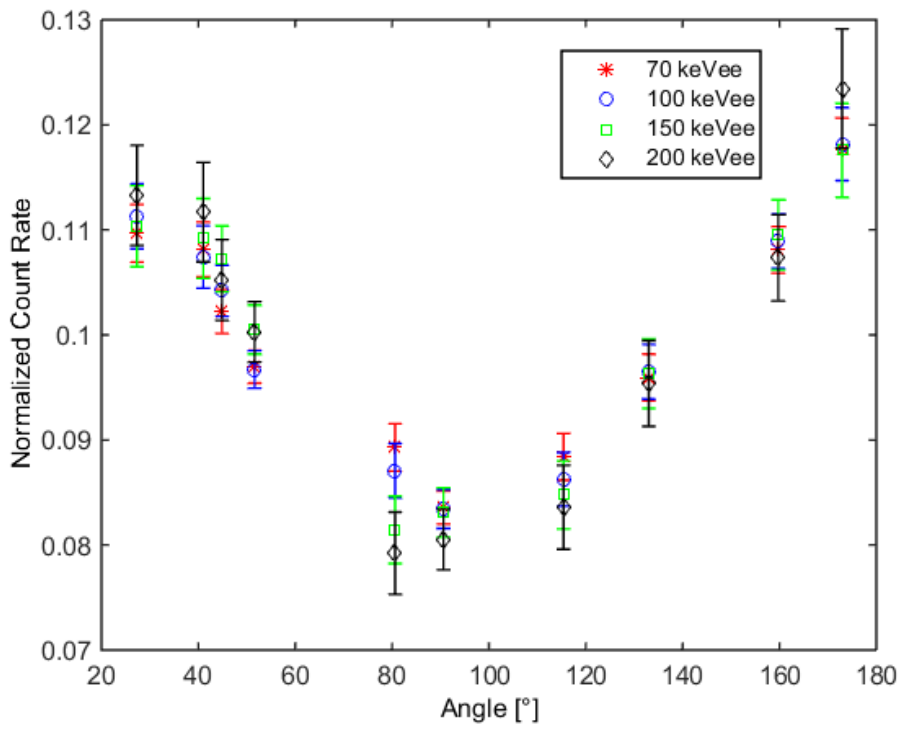


Figure 10: Experiment PM3 neutron-neutron cross-talk corrected coincidence angular distributions with a light output threshold varied over 70 to $200 \mathrm{keVee}$. Vertical error bars represent one standard deviation statistical uncertainty.

297

298 The neutron-neutron distributions for PM2 and PM3 show similar anisotropy, but the Cf-252 299 distribution is slightly more peaked toward 180 degrees. Slight differences in PM2 and PM3 size 300 and isotopic composition do not manifest in the neutron-neutron angular coincidence 301 distributions.

302 Figure 11 shows the ratio of 180 degree to 90 degree coincidences. As the light output 303 threshold increases, the observed neutron-neutron coincidences become more anisotropic. More 304 coincidences in the 90-degree pairs include low energy neutrons than in the 180-degree pairs; 305 thus, as threshold increases so does anisotropy. Greater anisotropy is expected because more 306 momentum is imparted to a neutron emitted near the fragment direction than to a neutron emitted 307 perpendicular to the fragment direction. The Cf-252 data shows much stronger anisotropy than 308 the $\mathrm{Pu}-240$ data, but the slope of increasing anisotropy is similar. The data for the two $\mathrm{Pu}-240$ 309 samples, PM2 and PM3, agrees within the statistical uncertainty of this experiment. 


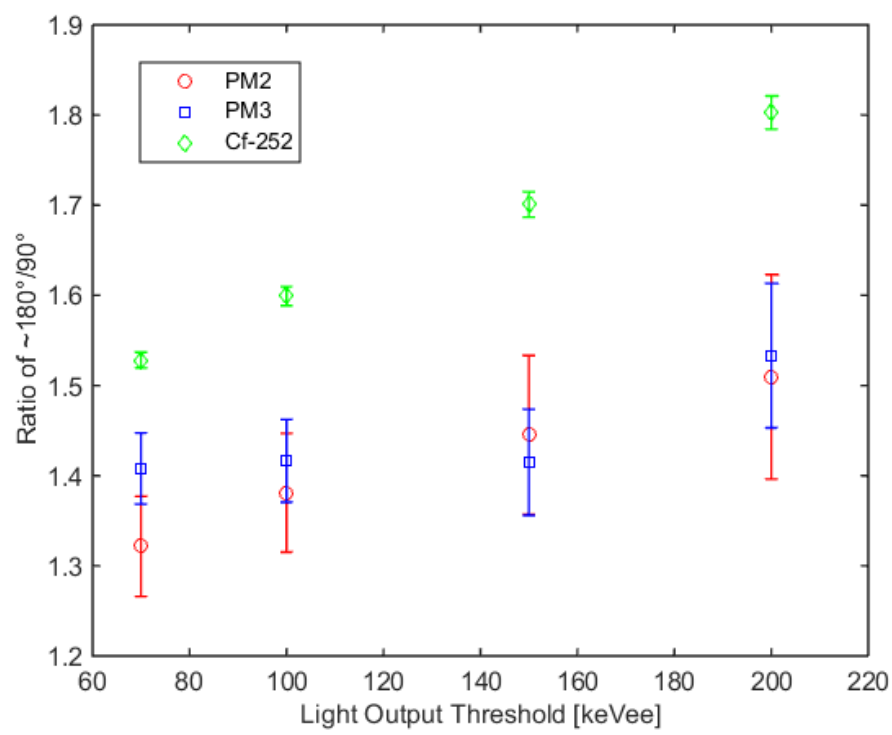

Figure 11: Experiment ratio of neutron-neutron coincidences at 180-degree to 90-degree detector pairs as a function of light output threshold.

\section{CONCLUSIONS}

Prompt neutron anisotropy from $\mathrm{Pu}-240$ was observed and quantified in laboratory experiments for the first time. The experiments were performed using organic liquid scintillators with two plutonium metal samples; the results agreed within statistical uncertainties. Cf-252 spontaneous fission neutrons above $0.65 \mathrm{MeV}$ were observed to be significantly more anisotropic than $\mathrm{Pu}-240$ spontaneous fission neutrons above that same energy. These experiment results could be used to improve prompt fission neutron models used in Monte Carlo codes for nonproliferation and safeguards applications.

MCNPX-PoliMi simulation of the PM3 laboratory and detector system reproduced experiment pulse height and cross-correlation distributions for 90-degree and 175-degree detector pairs. MCNPX-PoliMi simulations were used to estimate the cross-talk fraction and then to remove that fraction from the neutron-neutron angular distributions. More than $20 \%$ of small angle 
Future experiments with reduced uncertainty, especially in detector pair angles, are necessary to

We thank Dr. Enrico Padovani for his assistance in understanding the MCNPX-PoliMi fission models. We also

This research was performed under appointment to the U.S. Department of Energy Nuclear Nonproliferation International Safeguards Graduate Fellowship Program sponsored by the National Nuclear Security Administration's Office of Nonproliferation and International Security.

This work was funded in-part by the Consortium for Verification Technology under Department of Energy National Nuclear Security Administration award number DE-NA0002534 and by the Office of Defense Nuclear Nonproliferation Research \& Development (DNN R\&D), National Nuclear Security Administration, US Department of Energy.

\section{REFERENCES}

[1] N. Ensslin, W. C. Harker, M. S. Krick, D. G. Langner, M. M. Pickrell, J. E. Stewart, “Application Guide to Neutron Multiplicity Counting”, LA-13422-M, Los Alamos National Laboratory (1998); http://permalink.lanl.gov/object/tr?what=info:lanl-repo/lareport/LA-13422-M.

[2] P. Santi and M. Miller, "Reevaluation of Prompt Neutron Emission Multiplicity Distributions for Spontaneous Fission," Nucl. Sci. Eng., 160, 190 (2008); http://dx.doi.org/ 10.13182/NSE07-85.

[3] J. L. Dolan, M. Flaska, S. A. Pozzi, D. L. Chichester, "Passive measurements of mixed-oxide fuel for nuclear nonproliferation", Nucl. Instrum. Methods Phys. Res., Sect. A, vol. 703, pp. 102-108, (2013); http://dx.doi.org/10.1016/j.nima.2012.11.092.

[4] S. A. Pozzi, B. Wieger, A. Enqvist, S. D. Clarke, M. Flaska, M. Marcath, E. Larsen, R. C. Haight, and E. Padovani, "Correlated Neutron Emissions from Cf-252," Nuclear Science and Engineering, vol. 178(2), pp. 250-260, (2014).

[5] S. A. Pozzi, E. Padovani, "MCNP-PoliMi: A Monte Carlo Code for Correlation Measurements," Nucl. Instrum. Methods Phys. Res., Sect. A, vol. 513, no. 3, 618-623 (2003); http://dx.doi.org/10.1016/j.nima.2003.06.012.

[6] J. Randrup and R. Vogt, "Refined treatment of angular momentum in the event-by-event fission model FREYA," Phys. Rev. C, 89, 044601 (2014); http://dx.doi.org/10.1103/PhysRevC.89.044601.

[7] C. Wagemans, The Nuclear Fission Process, CRC Press, Boca Raton, Florida (1991).

[8] L. Holewa, W. Charlton, E. Miller, S. Pozzi, "Using neutron angular anisotropy to dynamically determine the ratio of the ( $\alpha, n)$ rate to spontaneous fission rate for coincidence counting applications," Nucl. Instrum. Methods Phys. Res., Sect. A, vol. 701, pp. 249-253 (2013); http://dx.doi.org/10.1016/j.nima.2012.11.026.

[9] J.S. Fraser, "The Angular Distribution of Prompt Neutrons Emitted in Fission," Phys. Rev., vol. 88, no. 3, pp. 536-541, (1952); http://journals.aps.org/pr/pdf/10.1103/PhysRev.88.536.

[10] H.R. Bowman, J.C.D. Milton, S.G. Thompson, W.J. Swiatecki, "Further Studies of the Prompt Neutrons from the Spontaneous Fission of Cf-252," Phys. Rev., vol. 129, no. 5, pp. 2133-2147, (1963); http://dx.doi.org/10.1103/PhysRev.129.2133.

[11] C. Wagemans, E. Allaert. A. Deruytter, R. Barthelemy, P. Schillebeeckx, "Comparison of the energy and mass characteristics of the ${ }^{239} \mathrm{Pu}\left(\mathrm{n}_{\mathrm{th}}, \mathrm{f}\right)$ and the ${ }^{240} \mathrm{Pu}(\mathrm{sf})$ fragments," Phys. Rev. C, vol. 30, no. 1, pp. 218-223, (1984); http://dx.doi.org/10.1103/PhysRevC.30.218.

[12] J. L. Dolan, M. Flaska, A. Poitrasson-Riviere, A. Enqvist, P. Peerani, D. L. Chichester, S. A. Pozzi, "Plutonium measurements with a fastneutron multiplicity counter for nuclear safeguards applications", Nucl. Instrum. Methods Phys. Res., Sect. A, vol. 763, pp. 565-574, (2014); http://dx.doi.org/10.1016/j.nima.2014.06.028.

[13] F.D. Brooks, R. W. Pringle, B. L. Funt, "Pulse Shape Discrimination in a Plastic Scintillator," Trans. Nucl. Sci., vol. 7, no. 2-3, pp. 35-38, (1960); http://ieeexplore.ieee.org/stamp/stamp.jsp?tp=\&arnumber=4315733.

[14] G. F. Knoll, Radiation Detection and Measurements, John Wiley \& Sons, Hoboken, New Jersey (2010).

[15] J. K. Polack, M. Flaska, A. Enqvist, C. S. Sosa, C. C. Lawrence, S. A. Pozzi, "An Algorithm for Charge-Integration, Pulse-Shape Discrimination and Estimation of Neutron/Photon Misclassification in Organic Scintillators,” Nucl. Instrum. Methods Phys. Res., Sect. A, vol. 795, pp. 253-267, (2015); http://dx.doi.org/10.1016/j.nima.2015.05.048. 
[16] S.A. Pozzi, M.M. Bourne, J.L. Dolan, K. Polack, C. Lawrence, M. Flaska, S.D. Clarke, A. Tomanin, P. Peerani, "Plutonium metal vs. Oxide determination with the pulse-shape-discrimination-capable plastic scintillator EJ-299-33," Nucl. Instrum. Methods Phys. Res., Sect. A, vol. 767, pp. 188-192, (2014); http://dx.doi.org/10.1016/j.nima.2014.08.002

[17] G. A. Petrov, A. S. Vorobyev, V. E. Sokolov, A. M. Gagarski, I. S. Guseva, O. A. Shcherbakov, V. I. Petrova, T. A. Zavarukhina, G. V. Val'ski, Yu. S. Pleva, T. E. Kuzmina, "Search for Scission Neutrons Emitted in Low Energy Fission of Heavy Nucleus," AIP Conf. Proc., 1175, 289 (2009); http://dx.doi.org/10.1063/1.3258239. 